\title{
Unusual Eccentricity and Inclination Distributions of Trans-Neptunian Objects and Trans-Neptunian Binaries
}

\author{
Robert B. Brown \\ Department of Astronautics, United States Air Force Academy, Colorado, USA
}

Email address:

robert.brown@usafa.edu

To cite this article:

Robert B. Brown. Unusual Eccentricity and Inclination Distributions of Trans-Neptunian Objects and Trans-Neptunian Binaries. International Journal of Astrophysics and Space Science. Vol. 6, No. 1, 2018, pp. 28-37. doi: 10.11648/j.ijass.20180601.13

Received: January 23, 2018; Accepted: February 3, 2018; Published: February 26, 2018

\begin{abstract}
The eccentricity and inclination of trans-Neptunian objects (TNOs) decrease significantly as radius of perihelion increases. Also, the percentage of TNOs in almost circular orbits with low inclinations increases dramatically with perihelion distance. These unusual distributions presented here cannot be explained by observational bias or the poorly determined orbits in the Kuiper belt. Instead, they may provide evidence about the origin of TNOs. Most theories are also unable to explain these strange orbital distributions. However, if TNOs passed close to Neptune because they migrated relatively quickly away from the Sun, their eccentricities and inclinations should both be inversely related to perihelion distance as shown. This TNO-migration theory can also help explain the origin of trans-Neptunian binaries (TNBs), and it accurately predicts the relationships between TNBs' separation distances and their heliocentric perihelia, eccentricities, and inclinations.
\end{abstract}

Keywords: Trans-Neptunian Objects, Trans-Neptunian Binaries, Kuiper Belt

\section{Introduction}

The Solar System has over 2300 known objects with a semimajor axis greater than Neptune's orbit of 30 AU. ${ }^{1}$ These trans-Neptunian objects (TNOs) may hold important clues about the formation and evolution of the Solar System. Over 2000 TNOs also have perihelion distances, $q$, greater than $30 \mathrm{AU}$, meaning they always remain beyond Neptune's orbit. Because these TNOs are less affected by the giant planets, their orbits in particular may provide evidence of the early formation of TNOs and possibly other bodies in the Solar System as well.

The unusual orbits of trans-Neptunian binaries (TNBs) may help identify the origin of TNOs. There are 70 known TNBs with $q>30$ AU [1]. For 26 of these pairs, their mutual orbits about each other are also known, which might provide useful information about how these bodies formed [2]. Two studies of the seven most widely separated TNBs concluded that these loosely bound objects, which they call ultra-wide TNBs, were not formed from collisions and never had a close encounter with a giant planet $[3,4]$. Such perturbations

1 Orbital parameters for this report were collected from the Jet Propulsion Laboratory's small-body database on 2017 January 2. would have stripped these delicate pairs from their orbits around each other.

It has been postulated that TNOs gently formed in situ from planetesimals that remained after the formation of the Solar System. However, at that distance from the Sun, the cloud of planetesimals would have been too dispersed to have coalesced [5]. It has also been proposed that TNOs may have formed in their present orbits from collisions, but these collisions would have been too infrequent, and they would have knocked the widest TNBs out of their binary orbits $[4,6,7]$.

Another theory postulates that TNOs formed closer to the Sun in orbits with small eccentricities $(e)$, low inclinations $(i)$, and semimajor axes less than 30 AU [8-14]. Then, during a proposed outward migration by Neptune, TNOs were scattered into their present orbits. This better explains how TNOs coalesced closer to the Sun, and it can also explain why so many TNOs are in resonance with Neptune today. Simulations show that Neptune's migration can explain some aspects of TNO orbits, but another unknown event likely also disturbed the Kuiper belt [13]. In addition, this Neptune-migration theory cannot explain how ultra-wide TNBs exist. If these fragile pairs received a large 
gravitational boost from Neptune, they would have been permanently separated from their shared orbits $[4,15,16]$. Neptune's migration is also unable to explain why there are so many cold TNOs (those with small eccentricities and low inclinations). If all of the TNOs were perturbed by Neptune, very few should be in nearly circular orbits near the ecliptic. To address some of these concerns, Levison et al. [14] proposed a modified model of Neptune's migration that could deliver TNOs to the Kuiper belt while maintaining relatively low inclinations, but their theory can still not explain the abundance of nearly circular objects in the classical Kuiper belt. It has also been proposed that primarily just those TNOs with large eccentricities and inclinations were scattered by Neptune, and the cold population of TNOs is a distinct group of bodies that likely had another unknown origin [17, 18].

A more recent study of TNOs also proposes that TNOs formed inside Neptune's orbit, and later many were perturbed by Neptune. This slightly different theory, however, postulates that TNOs came close to Neptune not due to Neptune's migration, but because the TNOs were spiraling away from the Sun [19]. According to this TNO-migration theory, as TNOs passed $30 \mathrm{AU}$, some developed a resonance with Neptune. Gravitational perturbations from Neptune also increased many of their eccentricities and inclinations. Other TNOs that spiraled away from the Sun relatively quickly were able to pass $30 \mathrm{AU}$ without coming close to Neptune. As a result, their orbits remained relatively undisturbed, and they are still in fairly circular orbits with low inclinations today. According to this TNO-migration theory, many TNOs continued migrating away from the Sun, increasing their perihelion distances beyond $30 \mathrm{AU}$, and eventually settled in the orbits observed today.

Every known study that proposes TNOs formed inside Neptune's orbit assumes they began in nearly circular orbits near the plane of the ecliptic $[8-11,13]$. If this is true, and if TNOs came close to Neptune, either due to Neptune's migration or the migration of TNOs, all of their eccentricities would have increased, and in many cases, their inclinations would have also increased. Once they were far beyond Neptune's orbit, their eccentricities and inclinations would not have changed significantly, and simulations show perturbations from the giant planets do not dramatically affect these orbital elements today. Therefore, a close examination of TNOs' eccentricities and inclinations may be beneficial in determining if they were perturbed by Neptune in the past and could lead to a better understanding of their origins.

This paper is organized as follows: Section 2 shows the unusual eccentricity and inclination distributions of TNOs as a function of perihelion distance, including an examination of potential biases. Section 3 discusses these results, and based on the proposed TNO-migration theory, some predictions are made concerning the perihelion distances, eccentricities, and inclinations of TNBs relative to their separation distances. Evidence supporting these predictions is then presented in Section 4, and the results are summarized in Section 5.

\section{Eccentricity and Inclination Distributions of TNOs}

\subsection{Distributions of All TNOs Beyond $30 \mathrm{AU}$}

TNOs are often classified based on their semimajor axes, and distributions of TNOs' eccentricities and inclinations are almost always presented as a function of semimajor axis. However, much more interesting details become apparent when these distributions are displayed as a function of radius of perihelion. For example, of the 2003 known TNOs with $q$ greater than $30 \mathrm{AU}$, slightly more than half (1072) have a radius of perihelion less than $39 \mathrm{AU}$. The average eccentricity for these TNOs is 0.25 , and their average inclination is $16.8^{\circ}$. In contrast, the 931 TNOs with $q$ greater than 39 AU have much smaller eccentricities and inclinations with average values of only 0.08 and $8.3^{\circ}$ respectively. The median eccentricities and inclinations of these two groups of TNOs are also dramatically different. TNOs with perihelia between 30 and 39 AU have a median eccentricity of 0.20 and a median inclination of $14.2^{\circ}$. In comparison, those TNOs with perihelion distances greater than 39 AU have a median eccentricity of just 0.08 and an inclination of only $3.5^{\circ}$.

These inverse relationships between radius of perihelion and both eccentricity and inclination are even more apparent when smaller intervals of perihelion are used beyond 39 AU. As shown in Table 1, both the average and median eccentricities decrease dramatically as the radius of perihelion increases. In general, the average and median inclinations also decrease significantly as perihelion distance increases.

Even more startling is the increase in the number and percentage of orbits that are almost circular (defined here as $e$ $<0.05)$ as their perihelion distances increase beyond 40 AU. For example, there are 1235 known TNOs with perihelion distances between 30 and $40 \mathrm{AU}$, yet only 42 of their orbits (or $3 \%$ ) have $e<0.05$. In contrast, there are 768 TNOs with $q>40$ $\mathrm{AU}$, and 376 of these (or $49 \%$ ) have an eccentricity smaller than 0.05 .

As shown in the top panel of Figure 1, this inverse relationship between perihelion distance and eccentricity is even more evident when smaller perihelion intervals are used. This plot, showing the percentage of TNOs with $e<0.05$, uses perihelion intervals of $1 \mathrm{AU}$ between 30 and $42 \mathrm{AU}$, and the far right column includes all TNOs with $q>42$ AU. Because the perihelion interval for this column is different, it is separated from the rest of the chart with a thin white line. Although some of the bodies in this interval have very large perihelion distances, over $80 \%$ of them have a radius of perihelion between 42 and $45 \mathrm{AU}$. The bottom panel of Figure 1 is a similar plot showing the percentage of TNOs with $i<5^{\circ}$ for each of these same perihelion intervals. The 13 numbers directly below each plot indicate how many TNOs in each interval have either $e<0.05$ or $i<5^{\circ}$, and the numbers at the very bottom of Figure 1 show that there are between 81 and 338 TNOs in each of these perihelion intervals, which is a sufficient number for comparisons. 
Table 1. Eccentricities and inclinations of TNOs as a function of perihelion distance.

\begin{tabular}{|c|c|c|c|c|c|}
\hline \multirow{2}{*}{ Range of $q(\mathrm{AU})$} & \multirow{2}{*}{ Population } & \multicolumn{2}{|l|}{ Eccentricity } & \multicolumn{2}{|c|}{ Inclination $\left({ }^{\circ}\right)$} \\
\hline & & Average & Median & Average & Median \\
\hline $30-39$ & 1072 & 0.25 & 0.20 & 16.8 & 14.2 \\
\hline $39-40$ & 163 & 0.14 & 0.11 & 13.1 & 9.8 \\
\hline $40-41$ & 201 & 0.09 & 0.08 & 7.5 & 4.3 \\
\hline $41-42$ & 229 & 0.07 & 0.06 & 6.5 & 3.0 \\
\hline$>42$ & 338 & 0.05 & 0.02 & 7.6 & 2.9 \\
\hline
\end{tabular}
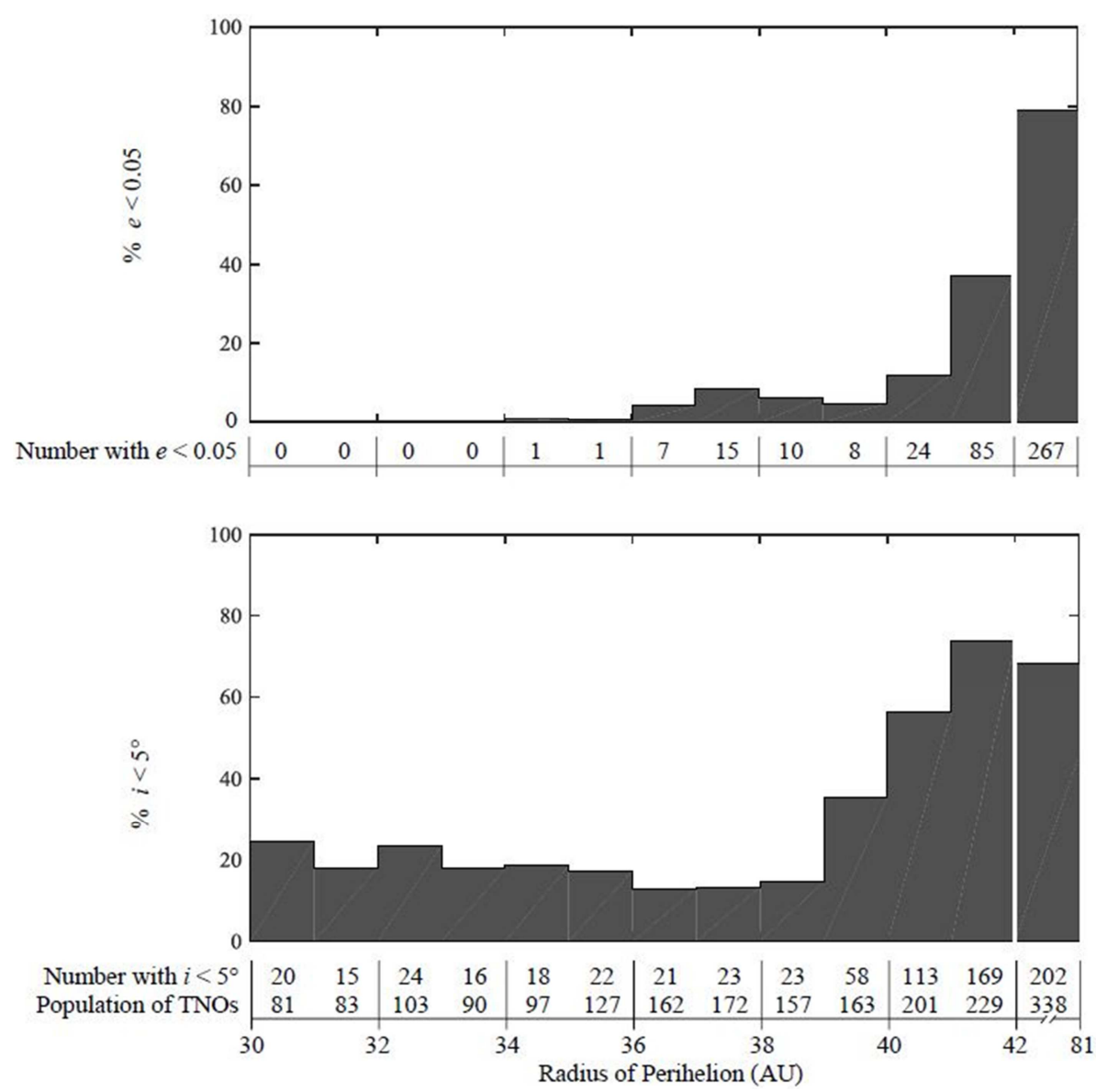

Figure 1. Number and percentage of TNO orbits with an eccentricity less than 0.05 (top) and an inclination less than $5^{\circ}$ (bottom) for various ranges of perihelion. As perihelion distances increase, many more TNOs have e $<0.05$ and $i<5^{\circ}$.

The top panel in Figure 1 shows that for the first four perihelion ranges, between $30 \mathrm{AU}$ and $34 \mathrm{AU}$, there are a total of 357 TNOs, yet not one of these bodies is in a nearly circular orbit with $e<0.05$. In fact, they all have eccentricities greater than 0.06 . For the following six perihelion intervals, between 34 and $40 \mathrm{AU}$, there are $878 \mathrm{TNOs}$ and only 42 of these (5\%) have an eccentricity less than 0.05 . The number and percentage of orbits that are nearly circular increase dramatically as perihelion distance increases beyond $40 \mathrm{AU}$. For example, 85 of the TNOs with perihelia between 41 and $42 \mathrm{AU}$ are almost circular, which is $37 \%$ of this population. Also, the far right column in the top panel of Figure 1 shows that 267 of the TNOs with $q>42$ AU are nearly circular, which is an astonishing $79 \%$ of these bodies.

The bottom panel of Figure 1 shows that as perihelion distances increase TNOs are also much more likely to have very low inclinations. For the $1072 \mathrm{TNO}$ orbits with perihelion distances between 30 and $39 \mathrm{AU}$, the percentage of orbits with $i<5^{\circ}$ are fairly constant, and only $17 \%$ of these orbits have this small of an inclination. In sharp contrast, these percentages begin to rise dramatically as perihelion distances increase beyond $39 \mathrm{AU}$.

Many TNO orbits have eccentricities less than 0.05 and also have inclinations less than $5^{\circ}$, meaning these bodies are in almost circular orbits and also remain near the plane of the ecliptic. Table 2 summarizes how the number and percentage of these orbits increase significantly as perihelion distance increases. As shown in the first row of Table 2, there are 1235 known TNOs with perihelia between 30 and $40 \mathrm{AU}$, yet only 12 of them have almost circular orbits with low inclinations, which is just $1 \%$ of this population. This percentage increases dramatically for every interval of perihelion distance beyond $40 \mathrm{AU}$. For example, the bottom row of Table 2 shows that there are 202 TNOs with $q>42 \mathrm{AU}$ that are almost circular and also remain near the ecliptic, which is $60 \%$ of these bodies. 
Table 2. TNOS with low eccentricities and inclinations as a function of perihelion distance.

\begin{tabular}{llll}
\hline Range of $\boldsymbol{q}$ & \multirow{2}{*}{ Population } & \multicolumn{2}{c}{$\boldsymbol{e}<\mathbf{0 . 0 5}$ and $\boldsymbol{i}<\mathbf{5}^{\circ}$} \\
\cline { 3 - 4 } (AU) & & Number & Percent \\
\hline $30-40$ & 1235 & 12 & 1 \\
$40-41$ & 201 & 11 & 5 \\
$41-42$ & 229 & 68 & 30 \\
$>42$ & 338 & 202 & 60 \\
\hline
\end{tabular}

\subsection{Potential Bias}

What could have caused these unusual eccentricity and inclination distributions? They might provide evidence about the origin of TNOs, but they also could be due to two potential sources of bias, both of which need to be addressed: (1) poorly determined TNO orbits and (2) observational bias.

TNOs with poorly determined orbits are often presumed to be circular. If they are detected near the ecliptic, they may also be assumed to have low inclinations. It is also reasonable that TNOs with larger perihelion distances are more likely to have poorly determined orbits and might therefore be incorrectly assumed to be in circular orbits with low inclinations. This could possibly explain the distributions presented in Section 2.1. These include all 2003 known TNOs with $q>30 \mathrm{AU}$, and many of these orbits are not well known. Each of these bodies has a condition code, also called an uncertainty parameter, which is a measure of the uncertainty of its orbit. This is a logarithmic scale from 0 to 9 , with 0 indicating the best-known orbits and 9 signifying the least well-known orbits. All of the known TNOs with $q>30$ AU have condition codes between 2 and 9 . It is possible that including those orbits that are not well defined might explain the unusual eccentricity and inclination distributions shown in Section 2.1. A simple way to test this potential bias is to remove the poorly defined orbits from these distributions. If these orbits are responsible for the unusual trends for eccentricity and inclination summarized in Figure 1 and Table 2, those trends should disappear when these bodies are excluded.

There are almost 700 known TNOs with condition codes of 6 or more. These are the least well-known orbits included in Section 2.1. When these poorly known orbits are excluded, there are 1309 remaining TNOs with $q>30$ AU and condition codes of 2 to 5 . Figure 2 shows the eccentricity and inclination distributions of these 1309 orbits. Similar to the previous plots, as perihelion distances increase, both the number and percentage of orbits that are almost circular increase, and so do the number and percentage of orbits with $i<5^{\circ}$. In addition, Table 3 shows that the number and percentage of orbits with both $e<0.05$ and $i<5^{\circ}$ also increase significantly with perihelion distance, just like the data in Section 2.1.
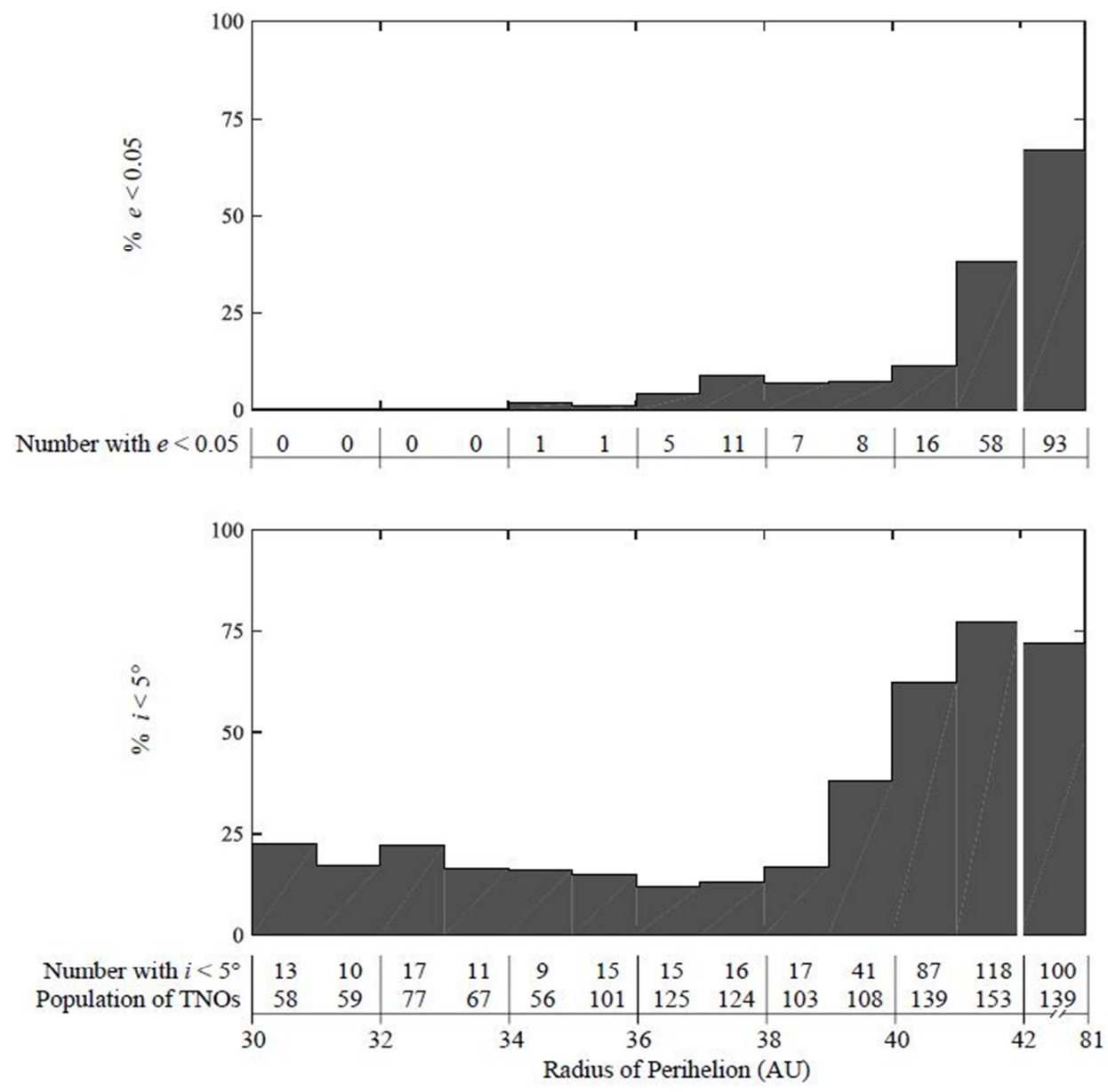

Figure 2. Number and percentage of TNO orbits with condition codes of 5 or less that have an eccentricity less than 0.05 (top) and inclination less than $5^{\circ}$ (bottom). Even after eliminating orbits that are not well defined (those with condition codes of 6 to 9), there are still significantly more TNO orbits with $e<$ 0.05 and $i<5^{\circ}$ as perihelion distance increases. 
Table 3. TNOs with condition codes of 5 or less with low eccentricities and inclinations.

\begin{tabular}{llll}
\hline Range of $\boldsymbol{q}$ & \multirow{2}{*}{ Population } & \multicolumn{2}{l}{$\boldsymbol{e}<\mathbf{0 . 0 5}$ and $\boldsymbol{i}<\mathbf{5}^{\circ}$} \\
\cline { 3 - 4 } (AU) & & Number & Percent \\
\hline $30-40$ & 878 & 9 & 1 \\
$40-41$ & 139 & 9 & 6 \\
$41-42$ & 153 & 50 & 33 \\
$>42$ & 139 & 82 & 59 \\
\hline
\end{tabular}

Even if only the well-known TNO orbits are included, these same trends for eccentricity and inclination are still clearly present. The best-known TNO orbits with $q>30$ AU have condition codes of 2 . Unfortunately, there are just 57 of these orbits, resulting in only zero to seven TNOs in each of the 13 perihelion ranges used in the previous figures. These small numbers do not allow for a good comparison. However, there are 655 TNOs with condition codes of 2 or 3 , resulting in 30 to 64 TNOs in each perihelion interval. The eccentricity and inclination distributions of these well-known TNO orbits are shown in Figure 3. Similar to the previous plots, the number and percentage of orbits with $e<0.05$ and $i<5^{\circ}$ increase significantly with radius of perihelion. In addition, Table 4 shows there are many more of these well-known orbits that are almost circular with low inclinations and large perihelion distances compared to those with smaller perihelia.
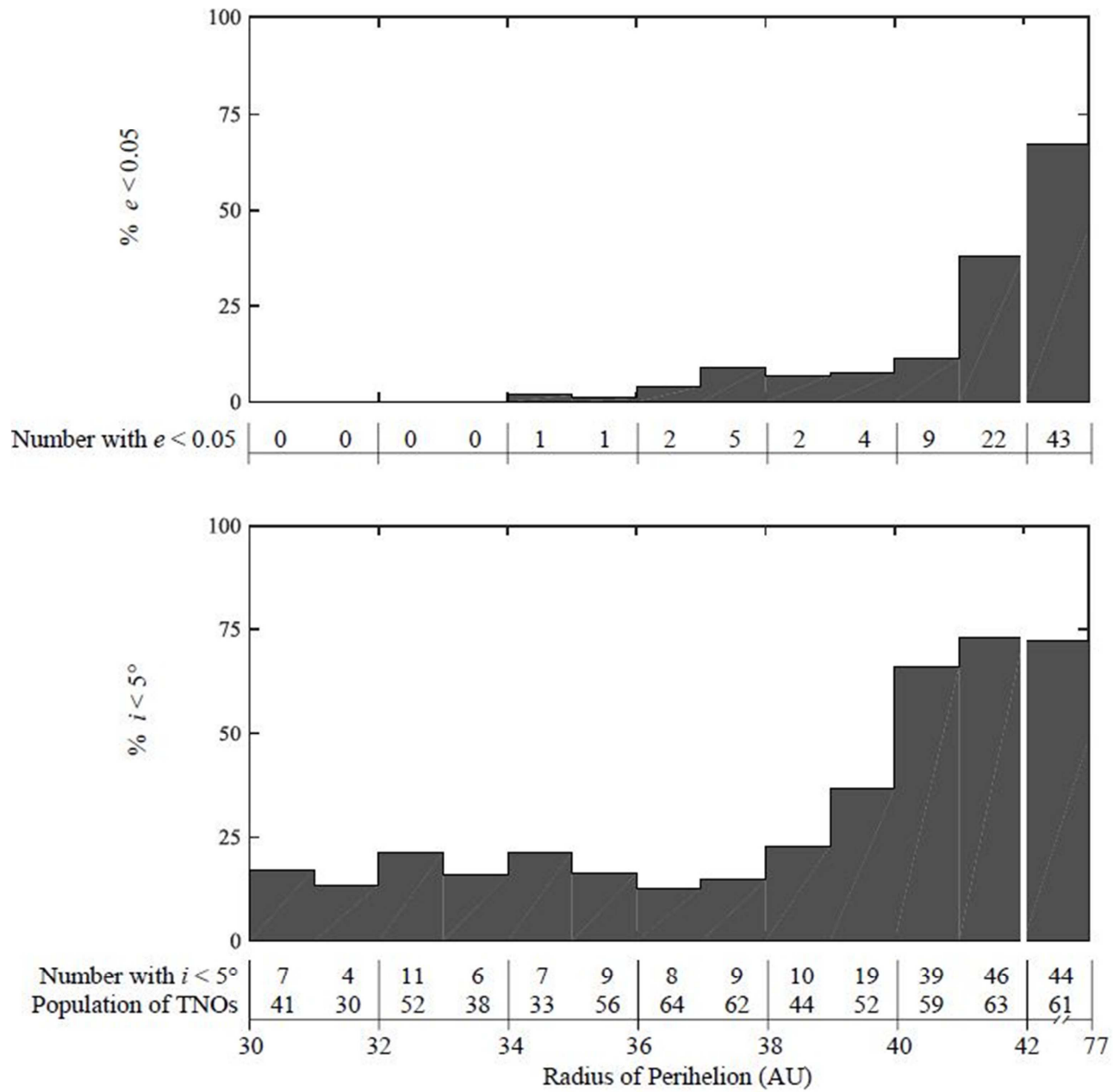

Figure 3. Number and percentage of the best-known TNO orbits that have an eccentricity less than 0.05 (top) and an inclination less than $5^{\circ}$ (bottom). Even for these well-known orbits, with condition codes of 2 or 3 , considerably more TNOs have e $<0.05$ and $i<5^{\circ}$ as perihelion distance increases.

Table 4. TNOs with condition codes of 3 or less with low eccentricities and inclinations.

\begin{tabular}{llll}
\hline Range of $\boldsymbol{q}$ & \multirow{2}{*}{ Population } & \multicolumn{2}{c}{$\boldsymbol{e} \mathbf{0 . 0 5}$ and $\boldsymbol{i}<\mathbf{5}^{\circ}$} \\
\cline { 3 - 4 } (AU) & & Number & Percent \\
\hline $30-40$ & 472 & 5 & 1 \\
$40-41$ & 59 & 6 & 10 \\
$41-42$ & 63 & 18 & 29 \\
$>42$ & 61 & 38 & 62 \\
\hline
\end{tabular}

Based on these results, it is extremely doubtful that the unusual eccentricity and inclination distributions of TNOs are due to poorly determined orbits. When the least well-known orbits are eliminated from the study, the distributions for eccentricity and inclination do not change. Also, even when only the best-known orbits are included in Figure 3 and Table 4 , the number of TNOs that have $e<0.05$ and $i<5^{\circ}$ still increases significantly with perihelion distance.

Could these unusual distributions of eccentricity and inclination instead be caused by observational bias? Although observational bias could explain some aspects of these distributions, it cannot account for the most significant 
characteristics shown in the previous figures and tables. In fact, observational bias would likely result in the opposite trends observed. For example, the top panel of Figure 1 shows that $79 \%$ of the TNOs with $q>42 \mathrm{AU}$ have $e<0.05$. This is an extremely high number, but considering the possible undiscovered TNOs, this percentage is likely too high. For a given perihelion distance, TNOs that are almost circular are easier to discover. In contrast, TNOs in highly elliptical orbits travel much farther from the Sun and spend a majority of their time near aphelion, so these objects are more difficult to detect. Therefore, there are likely many undiscovered elliptical orbits, and the actual percentages of TNOs in nearly circular orbits are probably less than what are shown in the previous figures. However, this is true for all ranges of perihelion, not just for those TNOs with large perihelion distances. So, although the percentages of orbits with $e<0.05$, shown in the top panels of Figures $1-3$, are probably too high, the general trend, that orbits with larger perihelion distances are more likely to be almost circular, is probably still accurate. In addition, observational bias cannot explain why not even one of the 357 TNOs with perihelia between 30 and 34 AU has a nearly circular orbit, and only 42 of the 878 orbits with $q$ between 34 and $40 \mathrm{AU}$ are almost circular. It is much easier to discover almost circular orbits than to detect elliptical orbits. So, why have only a few nearly circular orbits been discovered for these groups of TNOs? It is also easier to discover almost circular orbits at these distances from the Sun compared with TNOs that have larger perihelion distances, yet there are 376 TNOs with a radius of perihelion greater than $40 \mathrm{AU}$ that are almost circular. So, although observational bias likely resulted in artificially high percentages shown in the top panels of Figures $1-3$, it cannot explain why there are extremely few nearly circular orbits with perihelia between 30 and $40 \mathrm{AU}$ or the inverse relationship between radius of perihelion and eccentricity.

Observational bias is also unable to explain why the number and percentage of TNOs with an inclination less than $5^{\circ}$ increases with radius of perihelion, as shown in the bottom panel of the previous figures. Surveys near the ecliptic are common and biased toward discovering low-inclined objects that spend the majority of their time near the ecliptic. These surveys would certainly discover of a disproportionally large number of TNOs with $i<5^{\circ}$. However, these studies should find more TNOs with a low inclination that have a small perihelion distance compared with those that have a large radius of perihelion. So, observational bias would result in the exact opposite trend of what is presented in Figures 1-3.

Finally, it is also reasonable to expect that due to observational bias there should be more TNOs discovered with small perihelion distances that are in almost circular orbits and also have low inclinations compared with those that have a larger perihelion distances. This is the opposite of what is shown in Tables 2-4. For these reasons, it is doubtful that the strange distributions of eccentricity and inclination could be attributed to either poorly determined orbits or observational bias. Instead, these unusual distributions are most likely real, and they might, therefore, provide insight about the origin of TNOs.

\section{Discussion}

Most theories for TNOs' origins have difficulty explaining the inverse relationships between perihelion distance and eccentricity and inclination presented here. For example, it has been proposed that TNOs gently coalesced in their present orbits from planetesimals. However, assuming the planetesimals were evenly distributed, the expected number of TNOs with low eccentricities and inclinations should remain generally constant as perihelion distances increase, but this is clearly not the case. Also, other research shows that planetesimals would have been too dispersed to have coalesced this far from the Sun [5].

In contrast, the distributions of eccentricity and inclination could possibly be explained if TNOs formed in their present orbits as a result of collisions. If the concentration of bodies was initially greater between 30 and $40 \mathrm{AU}$, TNOs in this range may have experienced more collisions compared with those that have larger perihelion distances. These more frequent collisions could have increased the eccentricities and inclinations of TNOs with smaller perihelia. However, other research concluded that collisions would have been too infrequent for TNOs to form beyond $30 \mathrm{AU}$, and collisions would have permanently separated the widest TNBs $[4,6,7]$.

Another theory postulates that TNOs formed closer to the Sun, and they were later scattered into their present orbits as Neptune slowly migrated away from the Sun. As previously discussed, this theory can better explain how TNOs coalesced. It can also explain many aspects of TNO orbits, including the large number of eccentric and inclined orbits in the Kuiper belt and also why many TNOs are in resonance with Neptune. However, Neptune's migration cannot explain how ultra-wide TNBs exist. A large perturbation from Neptune would have pulled these fragile pairs out of their mutual orbits $[4,15,16]$. The Neptune-migration theory is also unable to explain why so many TNOs with large perihelion distances have very low eccentricities and inclinations. If all of the TNOs were scattered by Neptune, very few of these bodies should be in nearly circular orbits with low inclinations. Instead they should all be in elliptical orbits, and most, but not all, should also have inclinations greater than $5^{\circ}$. There should also not be any TNOs with perihelion distances beyond Neptune. A perturbation from Neptune would result in a maximum radius of perihelion of only $30 \mathrm{AU}$ (or $35 \mathrm{AU}$ if Neptune previously had an aphelion of roughly $35 \mathrm{AU}$ as speculated by Levison et al. [14]). However, there are over 1500 known TNOs with perihelion distances greater than $35 \mathrm{AU}$. To explain some of these problems, others believe that only some TNOs were scattered during Neptune's proposed migration, but it is still difficult to explain the origin of TNOs with large perihelion distances, small eccentricities, and low inclinations.

The TNO-migration theory, however, can explain both the unusual distributions of eccentricity and inclination shown in Section 2 as well as the existence of TNBs, including the ultra-wide TNBs. Similar to the theory that proposes 
Neptune's migration scattered the TNOs, the TNO-migration theory also postulates that TNOs were once inside Neptune's orbit in almost circular orbits near the ecliptic. Both theories also claim that some TNOs were later perturbed when they came close to Neptune. As a result, both of these theories can better explain how TNOs originally coalesced closer to the Sun, why so many TNOs are in resonance with Neptune, and why many TNOs have eccentric and inclined orbits. In contrast, however, the TNO-migration theory proposes that TNOs passed close to Neptune because the TNOs were moving away from the Sun, not because Neptune was migrating. This subtle difference is why the TNO-migration theory can explain the distributions of eccentricity and inclination presented here. This theory proposes that a majority, but not all, of the TNOs passed close to Neptune during their migration. For all of these TNOs, gravitational perturbations from Neptune would have increased their eccentricities and most of their inclinations. This would have also increased the velocities and semimajor axes of many of these bodies and flung them into the Kuiper belt. It is likely that other migrating bodies passed close to Neptune, but their velocities and semimajor axes decreased. So, they did not become TNOs, but may have become centaurs instead. Other TNOs, which presumably had larger non-gravitational forces, spiraled away from the Sun relatively quickly. This allowed many of these TNOs to avoid coming close to Neptune during their migration. As a result, they were able to maintain their nearly circular orbits with low inclinations. After the TNOs passed $30 \mathrm{AU}$, many of them, especially those that were spiraling away quickly, continued to migrate farther from the Sun, increasing their perihelion distances well beyond $30 \mathrm{AU}$. It is proposed that this is why there is an abundance of nearly circular orbits with very small inclinations and large perihelion distances. In contrast, those TNOs with smaller non-gravitational forces did not migrate quickly or travel very far. Therefore, almost all of these bodies were all perturbed by Neptune and their perihelia did not increase significantly beyond $30 \mathrm{AU}$. This explains why there are no TNOs with almost circular orbits and perihelia between 30 and $34 \mathrm{AU}$, and in general, there are very few nearly circular orbits with low inclinations and perihelion distances less than $40 \mathrm{AU}$.

This TNO-migration theory can also explain the existence of TNBs, even those with the widest separations. According to this theory, all of the TNOs, including the TNBs, were initially inside Neptune's orbit; then they began migrating away from the Sun due to yet unknown non-gravitational forces. As they moved farther from the Sun, their Hill spheres increased, which made it easier for them to capture a neighboring moon during their migration. It is also postulated that many of these pairs, especially the most widely separated TNBs, were some of the TNOs that had large non-gravitational forces. As a result, these pairs moved quickly past $30 \mathrm{AU}$, allowing some of them to avoid coming close to Neptune. Because they did not receive a large perturbation from Neptune, they were not separated from their moons and were also able to maintain their nearly circular heliocentric orbits close to the ecliptic. Also, because of their larger non-gravitational forces, their perihelion distances increased well beyond $30 \mathrm{AU}$ as they spiraled away from the Sun.

If this TNO-migration theory is correct, an average TNB should have a larger radius of perihelion, a smaller eccentricity, and a smaller inclination than a typical non-binary TNO. This should be especially true for the most widely separated TNBs, including all seven of the ultra-wide TNBs. Because these are very loosely bound pairs, they likely moved quickly past 30 $\mathrm{AU}$ and never came close to a giant planet during their migration. Because it is speculated that they quickly migrated beyond Neptune, they should all have large perihelion distances. They should also have small eccentricities and low inclinations because they were not perturbed by Neptune. For the other TNBs with known separation distances, the widest separated binaries should have larger perihelion distances and be more likely to be in nearly circular heliocentric orbits with low inclinations compared with non-binary TNOs. In comparison, some, but not all, of the TNBs with unknown separation distances may have also avoided Neptune. Therefore, on average these TNBs should also have a larger radius of perihelion and a smaller eccentricity and inclination than non-binary TNOs, but these differences should not be as great as the widest TNBs.

\section{Eccentricity and Inclination Distributions of TNBs}

There are 70 known TNB pairs with $q>30$ AU. All of them have condition codes of 5 or less, meaning their orbits around the Sun are fairly well known. For 26 of these pairs, their orbits about each other are also known [2, 3]. For these TNBs, a good measure of their separation distance is the ratio of the semimajor axis for their mutual orbit around each other, $a$, and their Hill radius, $R_{H}$. Parker et al. [3] characterized the seven widest binaries with $0.0809 \leq a / R_{H} \leq 0.2222$. Grundy et al. [2] reported on the mutual orbits for 19 other TNBs with $q>30$ AU, which are not as widely separated. Grundy et al. also compiled a complete list of TNBs for Lowell Observatory, which includes 44 other binaries with $q>30$ AU whose orbits about each other are not yet known [1]. The heliocentric orbits for all of these TNBs were compared with the 1239 non-binary TNOs that also have condition codes of 5 or less and have a radius of perihelion greater than $30 \mathrm{AU}$. These comparisons support all of the predictions made in the previous section.

The top panel of Figure 4 shows the perihelion distances for the 26 TNBs, with known mutual orbits, as a function of $a / R_{H}$. The seven ultra-wide TNBs are depicted with larger circles, and the other TNB pairs with known separation distances are represented with smaller dots. The average radius of perihelion for the 44 other TNBs, with $q>30 \mathrm{AU}$ and an unknown $a / R_{H}$, is shown with a dashed line, and the solid line indicates the average radius of perihelion for the 1239 non-binary TNOs with condition codes of 5 or better and $q>$ 30 AU. As expected, in general $q$ increases with $a / R_{H}$, and the widest TNBs have larger perihelion distances than more tightly bound TNBs. Also, all of the ultra-wide TNBs have 
very large perihelion distances, with an average of $42.5 \mathrm{AU}$ and a minimum of 41.1 AU. This is significantly larger than the average radius of perihelion for non-binary TNOs of just 37.7 AU (shown with the solid horizontal line in the top of
Figure 4). In addition, the $44 \mathrm{TNBs}$ with an unknown $a / R_{H}$ have an average radius of perihelion of $38.7 \mathrm{AU}$ (dashed line), which is $1 \mathrm{AU}$ greater than the non-binary TNOs.
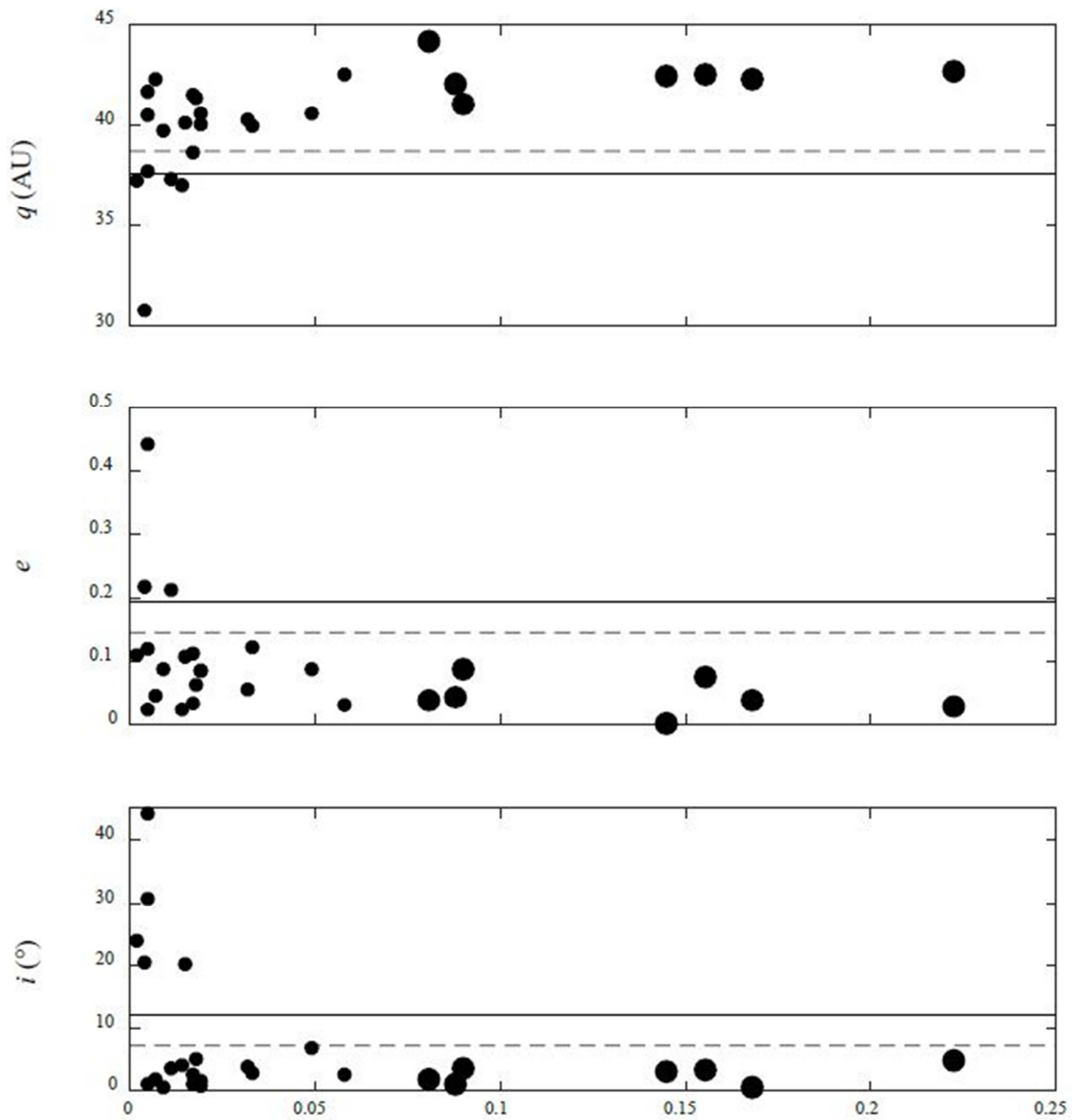

$a / R_{H}$

Figure 4. Heliocentric radius of perihelion (top), eccentricity (middle), and inclination (bottom) of TNBs as a function of their mutual semimajor axis divided by their Hill radius $\left(a / R_{H}\right)$. The seven ultra-wide TNBs are shown with large circles, and the smaller circles represent the other 19 TNBs with known values for $a / R_{H}$. The dashed lines show the average orbital parameters for the 44 other TNBs with an unknown a/R $R_{H}$ and the solid lines show the average values for all non-binary TNOs with similar condition codes and $q>30$ AU. As predicted, TNBs have larger perihelion distances, smaller eccentricities, and lower inclinations than non-binary TNOs, and these differences are more significant as $a / R_{H}$ increases.

The middle and bottom panels of Figure 4 show similar plots of eccentricity and inclination as functions of $a / R_{H}$. As predicted, mean eccentricity and inclination are inversely related to $a / R_{H}$, and the seven ultra-wide TNBs all have very small eccentricities and inclinations. Their eccentricities are all less than 0.09 , with an average of only 0.04 . They also all have inclinations less than $4.9^{\circ}$, with an average of only $2.6^{\circ}$. In addition, the TNBs with unknown $a / R_{H}$ have average eccentricities and inclinations that are less than the non-binaries, and these differences are not as great as the more widely separated TNBs.

Of the 26 TNBs shown in Figure 4 with known values for $a / R_{H}, 38 \%$ of their orbits are almost circular and also have $i<$ $5^{\circ}$. For the other 44 TNBs, with unknown values for $a / R_{H}, 23 \%$ are in orbits with $e<0.05$ and $i<5^{\circ}$. In comparison, only $10 \%$ of the 1239 non-binary TNO orbits with $q>30$ AU and condition codes of 5 or better are almost circular and also have an inclination less than $5^{\circ}$. These differences between binary and non-binary TNOs could indicate they had different origins. However, these differences can also be explained by the 
TNO-migration theory, and all of the data support the predictions from the previous section.

\section{Conclusions}

As TNOs' perihelion distances increase, their average and median eccentricities and inclinations decrease significantly. Furthermore, the percentages of TNOs in almost circular orbits with very low inclinations increase substantially with radius of perihelion. These trends are still clearly present when the poorest determined orbits are excluded and also when only the best-known orbits are considered. Therefore, these unusual distributions of eccentricity and inclination cannot be attributed to poorly determined orbits. They are also not due to observational bias. Instead, observational bias would probably result in the opposite trends. For these reasons, the inverse relationships between radius of perihelion and eccentricity and inclination appear to be real and without bias. Therefore, they may provide important evidence about the origin of TNOs.

Most theories do not explain the relationships between $q, e$, and $i$ shown in Section 2 and also how TNBs exist. If TNOs gently formed in situ from a cloud of planetesimals, their distributions of eccentricity and inclination should probably be uniformly distributed, yet Figures $1-3$ show this is clearly not the case. This theory also has difficulty explaining how TNOs would have coalesced so far from the Sun [5].

The eccentricity and inclination distributions covered in Section 2 could be explained, however, if TNOs formed from collisions. Assuming there were initially more bodies with small perihelia, it is reasonable that that these bodies would have experienced more collisions, which could have increased their eccentricities and inclinations. In contrast, those bodies with perihelion distances beyond $40 \mathrm{AU}$ might have had fewer collisions, and as a result may have been more likely to remain in nearly circular orbits near the ecliptic. However, other research shows that collisions would have been too infrequent beyond $30 \mathrm{AU}$ to have formed TNOs, and collisions would have separated the widest TNBs $[4,6,7]$.

Another theory postulates that TNOs formed closer to the Sun and were later scattered into their present orbits as Neptune migrated outward. This theory can better explain how TNOs originally coalesced and why so many TNOs are in resonance with Neptune today. Unfortunately, Neptune's migration cannot explain why there are hundreds of TNOs with large perihelion distances in almost circular orbits with low inclinations. If TNOs were perturbed by Neptune, they should be in eccentric orbits, and only a few should have small inclinations. This theory is also unable to explain why any TNO has a radius of perihelion greater than 30-35 AU or how ultra-wide TNBs survived a large gravitational perturbation from Neptune. To address some of these concerns, some believe that TNOs had multiple origins. Perhaps those with higher eccentricities and inclinations were perturbed during Neptune's migration, while those with smaller eccentricities and inclinations formed another way [18]. This eliminates some of the previous concerns, but it is still difficult to explain the formation of TNBs and the large number of TNOs with almost circular orbits and small inclinations well beyond Neptune.

However, if TNOs came in close contact with Neptune, not because of Neptune's migration, but rather because the TNOs were spiraling away from the Sun, all of these orbits can be explained. There would still be many TNOs in resonance with Neptune, and there would also be many eccentric and inclined orbits in the Kuiper belt. There could also have been some TNOs that had larger non-gravitational forces, which enabled them to quickly spiral past $30 \mathrm{AU}$ and avoid coming close to Neptune, allowing them to maintain their nearly circular orbits with small inclinations. Because of their larger non-gravitational forces, these TNOs would have also continued moving away from the Sun, and increased their perihelion distances well beyond $30 \mathrm{AU}$. This can explain why there are many TNOs with perihelia much greater than $30 \mathrm{AU}$ and also why considerably more of these bodies are in almost circular orbits with very small inclinations.

This TNO-migration theory can also explain the existence of TNBs. According to this theory, many of the TNBs, especially the most widely separated pairs, spiraled away from the Sun relatively quickly because they had larger non-gravitational forces. This increased their Hill spheres, helping them capture neighboring moons. This also allowed them to move quickly beyond $30 \mathrm{AU}$; so many were able to avoid coming close to Neptune. Based on this hypothesis, a number of predictions were made about the relationships between TNBs' separation distances and their heliocentric $q, e$, and $i$. All of these predictions were confirmed in Section 4. The ultra-wide TNBs all have large perihelion distances with very small heliocentric eccentricities and inclinations. The other TNBs also have larger perihelion distances, smaller eccentricities, and lower inclinations than non-binary TNOs, and these differences are more significant as $a / R_{H}$ increases. It is possible that these differences between binary and non-binary TNOs indicate separate origins for these two groups of TNOs. However, the distributions of both TNBs and non-binary TNOs can be explained by the TNO-migration theory.

If this theory is correct, new discoveries should confirm the trends presented here for the eccentricity, inclination, and radius of perihelion of TNOs. Also, future characterizations of TNBs should continue to show that as $a / R_{H}$ increases, the average radius of perihelion also increases, while eccentricity and inclination decrease. Additional research is also needed to identify the non-gravitational forces that may have moved TNOs away from the Sun. Although such a force would be much smaller than required for Neptune's proposed migration, there is no known force that can explain how TNOs migrated outward. This is a critical issue that needs to be addressed. Previous research speculated that the Yarkovsky effect could have been involved, but also acknowledged that this force by itself is too small to have moved large bodies beyond Neptune [19]. Identifying a larger non-gravitation force, which could have caused TNOs to migrate and modelling such a force in a large Monte Carlo study, is an important area of ongoing research. 


\section{Acknowledgements}

This document is for information only. No U.S. Government commitment to sell, loan, lease, co-develop, or co-product defense articles or provide defense services is implied or intended.

\section{References}

[1] W. M. Grundy et al., unpublished. $\mathrm{http}: / / \mathrm{www} 2$. lowell.edu/ grundy/tnbs/.

[2] "Five new and three improved mutual orbits of transneptunian binaries," Icarus, Vol. 213, No. 2, pp. 678-692 (2011).

[3] A. H. Parker et al., "Characterization of seven ultra-wide trans-neptunian binaries," ApJ, Vol. 743, No. 1, p. 1 (2011).

[4] A. H. Parker and J. J. Kavelaars, "Collisional evolution of ultra-wide trans-Neptunian binaries," ApJ, Vol. 744, No. 2, p. 139 (2011).

[5] M. D. Lemonick, "Pluto and beyond." Sci. Am., Vol. 311, No. 5, p. 46 (2014).

[6] P. Goldreich and Y. Lithwick, "Formation of Kuiper-belt binaries by dynamical friction and three-body encounters," Nature, Vol. 420, No. 6916, pp. 643-646 (2002).

[7] S. J. Weidenschilling, "On the origin of binary transneptunian objects,” Icarus, Vol. 160, No. 1, pp. 212-215 (2002).

[8] R. Malhotra, "The origin of Pluto's peculiar orbit," Nature, Vol. 365 , No. 6449, p. 819 (1993).

[9] M. J. Duncan, H. F. Levison, and S. M. Budd, "The dynamical structure of the Kuiper belt," AJ, Vol. 110, p. 3073 (1995).
[10] Q. Yu and S. Tremaine, "The dynamics of Plutinos," AJ, Vol. 118, No. 4, p. 1873 (1999).

[11] E. I. Chiang and A. B. Jordan, "On the Plutinos and Twotinos of the Kuiper belt," AJ, Vol. 124, No. 6, p. 3430 (2002).

[12] H. F. Levison and A. Morbidelli, "The formation of the Kuiper belt by the outward transport of bodies during Neptune's migration," Nature, Vol. 426, No. 6965 pp. 419-421 (2003).

[13] J. M. Hahn and R. Malhotra, "Neptune's migration into a stirred-up Kuiper belt: A detailed comparison of simulations to observations," AJ, Vol. 130, No. 5, p. 2392 (2005).

[14] H. F. Levison, A. Morbidelli, C. VanLaerhoven, R. Gomes, and K. Tsiganis, "Origin of the structure of the Kuiper belt during a dynamical instability in the orbits of Uranus and Neptune," Icarus, Vol. 196, No. 1, pp. 258-273 (2008).

[15] J. L. Margot, "Astronomy: Worlds of mutual motion," Nature, Vol. 416, No. 6882, pp. 694-695 (2002).

[16] J. M. Petit et al., "The extreme Kuiper belt binary 2001 QW322,” Science, Vol. 322, No. 5900, pp. 432-434 (2008).

[17] J. M. Petit et al., "The Canada-France Ecliptic Plane Survey - full data release: the orbital structure of the Kuiper belt," AJ, Vol. 142, No. 4, p. 131 (2011).

[18] A. Morbidelli, H. S. Gaspar, and D. Nesvorny, "Origin of the peculiar eccentricity distribution of the inner cold Kuiper belt," Icarus, Vol. 232, pp. 81-87 (2014).

[19] R. B. Brown and J. A. Firth, "Analysis of trans-Neptunian objects and a proposed theory to explain their origin," MNRAS, Vol. 456, No. 2, pp. 1587-1594 (2015). 